\title{
COLGAJOS LOCALES Y PEDICULADOS EN EL MANEJO DE LAS COMUNICACIONES Y FÍSTULAS OROANTRALES
}

\author{
Alexander Xavier Galvis Aparicio OD. ${ }^{1}$ \\ ${ }^{1}$ Residente del programa cirugía oral y maxilofacial, Universidad Militar Nueva Granada, Bogotá, Colombia.
}

\section{Resumen}

El manejo convencional de las comunicaciones oroantrales ha sido a través de técnicas quirúrgicas locales que a través del tiempo aun siguen vigentes para el manejo primario de dicha complicación. La mejoría del conocimiento de la anatomía detallada de la región maxilofacial ha introducido nuevas técnicas con el objetivo de mejorar e incluso tener nuevos alcances con respecto al manejo de las comunicación y fistula oro antrales que con las técnicas clásicas no se lograban. Esta revisión de tema muestra cada una de las opciones disponibles para el manejo de las comunicaciones y fistulas oro antrales con sus indicaciones, técnica quirúrgica y complicaciones.

Palabras clave: Comunicación oroantral, fistula oroantral, colgajo local.

\section{LOCAL AND PEDICULATED FLAPS IN THE MANAGEMENT OF OROAN- TRAL COMMUNICATIONS AND FISTULAS}

\begin{abstract}
Summary
The conventional management of oroantral communications has been performed through local surgical techniques which over time are still in vigor for the primary management of that complication. The improvement in the understanding of the maxillofacial region detailed anatomy has leaded to the introduction of new techniques with the aim of improving and even obtaining new achievements regarding the management of oroantral communication and fistulas which were not accomplished with the classical techniques. This subject review shows each one of the available options for the management of oroantral communications and fistulas with their indications, surgical techniques and complications.
\end{abstract}

Key words: Oroantral communication, oroantral fistula, local flap.

\section{RETALHOS LOCAIS E PEDICULADOS NO TRATAMENTO DAS COMUNICAÇÕES E FISTULAS OROANTRAIS}

\begin{abstract}
Resumo
O tratamento convencional das comunicações oro-antrais tem sido através de técnicas cirúrgicas locais que através do tempo ainda continuam vigentes para o tratamento primário dessa complicação. A melhoria do conhecimento da anatomia detalhada da região maxilo facial introduziu novas técnicas com o objetivo de melhorar e inclusive ter novos alcances com respeito ao tratamento das comu-
\end{abstract}

Correspondencia: Alexander Galvis alxagapa@hotmail.com. Dirección postal: Servicio de cirugía oral y maxilofacial, Hospital militar central, Tr. 3 No 49-00, Bogotá, Colombia.

Recibido: octubre 17 de 2011 Aceptado: diciembre 6 de 2011 
nicações e fistula oro-antrais que com as técnicas clássicas não era possível. Esta revisão de tema mostra cada uma das opções disponíveis para o tratamento das comunicações e fistulas oro-antrais com suas indicações, técnicas cirúrgicas e complicações.

Palavras chave: comunicação oro-antral, fistula oro-antral, retalho local.

\section{Introducción}

La comunicación oroantral (COA) se define como el espacio creado de manera accidental, iatrogénica, patológica o traumática entre la cavidad oral y el antro maxilar. Si la comunicación no es tratada dentro de las primeras 72 horas, puede cicatrizar de manera defectuosa y dará como resultado una fistula oroantral (FOA), y además un proceso infeccioso sinusal agudo $(1,2,3)$.

\section{Epidemiología}

Se ha estimado que la frecuencia de comunicaciones oroantrales en relación a la exodoncia del 1er molar superior es de 1 por cada 180 exodoncias, sin embargo aún permanece inexacto el porcentaje de comunicaciones oroantrales debido a que muchas no se diagnostican debido a su cicatrización espontanea. Además cabe recalcar el mayor número de exodoncias realizadas hoy en día sobre el tercer molar por la demanda en los tratamiento ortodonticos que del 1er molar superior, por lo que dicho diente puede tener una mayor morbilidad en la génesis de las COA $(1,4)$. Esta incidencia de las COA del $5 \%$ dada por la exodoncia de los molares superiores está determinada por la relación estrecha que existe entre sus raíces dentales y el antro maxilar, en donde hay una distancia entre $1-7 \mathrm{~mm}$, siendo el segundo molar superior el más cercano $(1,2,5,6)$, como ya se empezaba a dilucidar es las primeras anotaciones anatómicas realizadas porLeonardo Da Vinci en 1489 y Nathaniel Higmore en su Corporis Humani Disquisitio Anatomica en 1651 y (7).

Aunque el factor etiológico más común de las COA es la extracción de los molares superiores, no es el único, existen otros como la fractura de la tuberosidad del maxilar, osteomielitis, radioosteonecrosis, osteonecrosis por bisfosfonatos, órganos dentales dentro del seno maxilar, fracturas faciales que involucren apófisis alveolar, heridas por armas de fuego, tumores que involucren el antro maxilar, quiste adyacentes al seno maxilar, elevaciones del seno maxilar, e implantes dentales $(5,7)$.

Se ha reportado una mayor incidencia de COA en la tercera década, pero es en la 5ta década en donde se encuentra el mayor grado de complicaciones debido a una respuesta de cicatrización disminuida inherente al estadio de su vida que compromete la viabilidad de los tratamientos quirúrgicos instaurados (1).

\section{Diagnóstico}

Dentro de los signos propios de la COA encontramos regurgitación de líquidos hacia las fosas nasales, desinserción de prótesis, extrusión de pólipos sinusales, y con respecto a los signos de sinusitis maxilar normalmente relacionada con una FOA se encuentran descarga nasal, obstrucción nasal, halitosis, cacogueusia, cacosmia, voz nasal y dolor facial(8).

Anteriormente se utilizaba el sondaje como método diagnóstico, actualmente el examen clínico, el test de valsalva, y el examen radiológico son suficientes para determinar la existencia de una $\operatorname{COA}(4,9)$.

Con respecto al examen radiológico se ha establecido las proyecciones de Water's y panorámica como coadyuvantes para determinar la condición del seno maxilar y el desplazamiento de raíces, pero no para determinar la existencia de COA o FOA(4). Esto es importante ya que para el manejo del cierre de los defectos es primordial determinar la condición del seno maxilar y así poder establecer el entorno óptimo para que ocurra la cicatrización adecuada. Se ha reportado que una COA no tratada causa sinusitis aguda en 60\% de los casos al 3er día y es responsable del 10-12\% del total de las sinusitis crónicas del seno maxilar (10). Por ende su diagnóstico debe ser reconocido inmediatamente para evitar dichas complicaciones. El manejo del seno maxilar puede ser de manera conservadora con fármaco terapia antibiótica, descongestionante nasal y lavados, o quirúrgica según sea el caso (4). 
Se ha establecido que el manejo inmediato de las COA dentro de las primeras 24-48 horas tiene una tasa de éxito del 95\%, mientras que el cierre secundario de pasado este tiempo tiene un éxito del $67 \%(5,11,12)$. Las COA se ha clasificado según su tamaño en pequeñas $1-2 \mathrm{~mm}$, medianas $3-5 \mathrm{~mm}$ y largas $>5 \mathrm{~mm}$. Según su posición y extensión en la región maxilar puede ser descrita como anterior o posterior, vestibular, alveolar o palatina y con extensión a estructuras cercanas como la cavidad orbitaria. Esto es muy importante en la hora del planeamiento quirúrgico ya que determinara la viabilidad del colgajo que se utilice para realizar su tratamiento de forma quirúrgica $(5,13)$.

\section{Tratamiento}

Parece ser que Thomas Bell en 1829 en su libro Anatomy, Phisiology and diseases of the teeth, es el primero en describir un método algo similar al colgajo marginal para asistir el cierre de una COA (7).

Se ha determinado por evidencia clínica de que las COA menores de $4 \mathrm{~mm}$ cicatrizan espontáneamente, siempre y cuando no haya patología concomitante del seno maxilar o el alveolo dental $(1,5)$. Aquellas que son mayores a $4 \mathrm{~mm}$ debe realizar un planeamiento quirúrgico que incluye determinar la presencia de infección sinusal, tamaño de la lesión, tiempo de evolución del defecto y finalmente los manejos previos para observar el tejido disponible y siempre pensar en la posibilidad de colocación e implantes dentales a futuro $(1,6,14)$.

Anteriormente el manejo de las sinusitis maxilares se realizaban con el abordaje Caldwell-Luc con antrostomia del meato inferior, el mejor entendimento de la fisiología del complejo osteomeatal y su movimiento mucociliar hacia al ostium, ha llevado a que el manejo de la infección rino sinusal se realicen a través de cirugía endoscópica funcional (3). Además este tipo de abordajes ha traído inherentemente una mejor expectativa con respecto a la futura rehabilitación dental de la zona (15).

En aquellas COA mayores a $4 \mathrm{~mm}$ que no se les realiza manejo adecuado primario durante las primeras 3 semanas o las COA menores de $4 \mathrm{~mm}$ con patología adyacente (alveolo dental o seno maxilar), no se establecerá una cicatrización adecuada y por ende se desarrollara un epitelización unificada entre el epitelio pseudoestratificado ciliado columnar del seno maxilar y el epitelio escamoso estratificado queratinizado de la mucosa oral, el cual formara una FOA crónica que necesitara obligatoriamente un manejo quirúrgico (5).

Se han utilizado diferentes técnicas para el cierre de las COA y las FOA, desde las técnicas clásicas como la alveoloplastia interseptal (16), y otras muchas innovadoras como el uso de injerto autologo ya sea de mentón (17), región retromolar, cigoma o cresta iliaca a presión (10),injerto de cartílago auricular (18), regeneración tisular guiada, materiales sintéticos como oro, aluminio (19), polimetilmetacrilato, hidroxiapatita, análogos radiculares (20), xenoinjertos de colágeno (21), aloinjertos de fibrina (22), dura y otras más. Todas estas técnicas han surgido no solo con el objetivo de hacer el cierre definitivo de las de COA y FOA y evitar las complicaciones quirúrgicas inherentes a las técnicas clásicas sino también para obtener paralelamente la regeneración ósea del defecto residual y así tener una mejor opción terapéutica para la rehabilitación con implantes dentales a futuro. Incluso se ha reportado con el objetivo de rehabilitación inmediata el uso de autotrasplantes del tercer molar (23).Sin embargo es aun de preferencia para el manejo primario de las COA y FOA por los cirujanos el uso de los métodos clásicos como los colgajos locales con sus variaciones en el tiempo, entre ellos el colgajo vestibular de Rehrmann y el colgajo palatino de Ashley por su tasa de éxito y sobre todo su facilidad de realización. Con respecto al manejo secundario o lesiones extensas los colgajos a distancia como el de lengua, bolsa adiposa Bichat, miomucoso de buccinador y finalmente de musculo temporal son las opciones quirúrgicas disponibles $(4,11)$.

A pesar de la facilidad de la realización colgajos locales para el manejo primario de las COA por parte de clínicos con entrenamiento quirúrgico, esta complicación normalmente se presenta en la práctica del odontólogo general sin experiencia en el manejo quirúrgico primario y por ende el manejo es referido a una segunda estancia donde seguramente ya habrá como complicación una FOA y una sinusitis maxilar. Es por eso que métodos alternativos de manejo primario como aloinjertos y xenoinjertos deben constituirse como una herramienta importante dentro su práctica diaria (12).

Independiente de la técnica quirúrgica a utilizar se deben tener en cuenta 2 principios básicos, El primero, el seno maxilar y el alveolo dental debe estar 
libre de cualquier proceso infeccioso, el segundo, debe obtenerse un colgajo libre sin tensión de base amplia, bien vascularizado y que este sobre tejido óseo sano (2, $5,24)$. También se ha establecido como un principio quirúrgico según la viabilidad del tejido en el manejo de las lesiones fistulizadas restablecer el plano mucosa sinusal, el plano óseo y plano mucosa oral $(8,25)$.

\section{Colgajos locales}

Aunque se han descrito diferentes técnicas quirúrgicas para el cierre de las COA y las FOA, siempre se tendrá que encaminar el manejo desde la prevención con técnicas quirúrgicas pulcras, cierre inmediato con mucosa gingival para asegurar un coágulo sanguíneo y evitar riesgo infeccioso diferente al inherente al procedimiento quirúrgico en si, esto puede ser suficiente para el manejo de COA entre 1-4mm (1).

\section{El colgajo vestibular}

Se ha usado como primera opción es el colgajo vestibular de avance recto de Rehrmann de 1936, ya que es de fácil realización y manipulación, puede extenderse $1 \mathrm{~cm}$ más con incisiones relajantes periosticas, y tiene una gran irrigación (1). Se ha usado para cierre de COA y FOA menores de $1 \mathrm{~cm}$. Este colgajo tuvo una modificación por Berger en 1939 en donde las incisiones vestibulares se extienden hasta la mucosa yugal con el objetivo de lograr una mayor extensión del mismo (5).

La técnica quirúrgica se inicia con la infiltración submucosa con lidocaína $2 \%$ con epinefrina, posteriormente se realiza incisiones verticales vestibulares a nivel de los dientes adyacentes a las lesión para el levantamiento de un colgajo mucoperiostico, dado el caso de la necesidad de extensión del colgajo para posicionarlo sin tensiones se realiza relajantes periosticas o extender las relajantes verticales incluyendo lo necesitado de mucosa yugal. Finalmente se avanza y posiciona el colgajo sobre el defecto y se fija con sutura absorbible (20).

Dentro de las ventajas del colgajo destaca su fácil realización y alta irrigación, además con una tasa de éxito del 93\%. Sus desventajas radica en la perdida de la profundidad del surco vestibular que según Wowern 1982 es en el 50\% de los casos, esto afectando el pronóstico de rehabilitación a futuro, también se le atribuye ser un colgajo delgado que puede desgarrarse fácilmente durante su manipulación (6).

\section{El colgajo palatino}

Descrito por primera vez por Welty en 1920 y Ashley en 1939 describe su utilización en el cierre de una FOA (5).Aunque existen diversas modificaciones de este colgajo a través del tiempo, estas siguen basando su irrigación en la arteria palatina mayor (1). El único referente diferente fue establecido por Salinas en 1996 donde utilizo un colgajo palatino de base anterior y por Lee en 2002, teniendo en común la ligación de la arteria palatina mayor. Lee baso la irrigación del colgajo en el estudio realizado por McCarthy en 1995 donde establecen una anastomosis entre las arterias palatina ascendente y la palatina mayor(14), y Salinas sobre la hipótesis de una irrigación retrograda dada por la arteria nasopalatina y las anastomosisentre ramas de las arterias palatinas mayores a través de la línea media palatina. Sin embargo el éxito reportado por Lee fue del 76.2\%(26). Las técnicas basadas en el colgajo irrigado por la arteria palatina mayor basaron sus modificaciones en las complicaciones que el colgajo original presentaba como eransu difícil manipulación, el dolor posoperatorio importante dado por el paladar denudado del sitio donante y el riesgo de comprometer la perfusión sanguínea del colgajo al hacer la rotación del mismo. Una de las modificaciones fue realizada en 1974 por Takahashi en donde realiza un colgajo de isla palatina anterior separando la mucosa palatina queratinizada de la submucosa y el periostio, esto dando como resultado la protección del hueso palatino del lecho donante y el posible mejoramiento de la sintomatología dolorosa posoperatoria, sin embargo comprometiendo la vascularización del colgajo al no incluir la arteria palatina dentro de la isla. En 1985 Yamazaki realiza este mismo colgajo en isla anterior pero haciendo la disección del colgajo supraperiostico e incluyendo la arteria palatina mayor y asegurando la viabilidad del mismo. Con respecto al pliegue que se forma en la región medial del colgajo al hacer la rotación, el cual compromete la vascularización del mismo, Krueger propone realizar un seccionamiento en $\mathrm{V}$ de este pliegue, el cual Kale considera que atenta de igual forma con la vascularización del colgajo por lo cual propone extender la incisión medial de forma circular hasta la región de la tuberosidad $(9,14)$.

La técnica quirúrgica del colgajo palatino de avance y rotación sigue siendo la más utilizada, esta se inicia con la infiltración submucosa con lidocaína $2 \%$ con epinefrina, se diseña el colgajo determinando su 
extensión según el tamaño de la lesión, y siempre dejando $1 \mathrm{~cm}$ más de extensión en el diseño para evitar tensión en el momento de su posicionamiento, con respecto a los limites en el ancho están dados a $2-3 \mathrm{~mm}$ del rafe medio palatino y en la parte medial dejar $5 \mathrm{~mm}$ de encía marginal palatina con el fin de evitar algún daño periodontal. Posteriormente se hace el levantamiento del colgajo subperiostico se realiza la rotación y posicionamiento, finalmente se sutura con material absorbible. Se da manejo antibiótico y antiinflamatorio vía oral preoperatoriamente 1 hora antes y posoperatoriamente por 5 días, y enjuagues orales por 2 semanas con clorhexidina $0.12 \%$ (27).

Aunque se considera en la literatura de forma general como la segunda opción después del colgajo vestibular en el manejo de las COA y FOA, algunos autores lo consideran como la primera en los casos donde las características de la lesión apliquen para su uso, atribuyendo las ventajas de ser un colgajo con gran irrigación dada principalmente por la arteria palatina mayor y secundariamente por ramas colaterales provenientes de la arteria palatina ascendente, la arteria faríngea ascendente y anastomosis entre dichas ramas, un colgajo mucho más estable dado por su queratinización y grosor en comparación al colgajo vestibular y principalmente por su ubicación la no perdida de la profundidad del surco vestibular $(5,9,14,27)$.

\section{La bola adiposa de Bichat}

Descrita por primera vez por Heister en 1732 y Winslow en 1753 (28), sin embargo debe su nombre a Xavier Bichat quien reporta su constitución adiposa en 1801. Scammon en 1919 describe brevemente las características anatómicas de la bola adiposa Bichat. Egyedi en 1977 y Tideman 1986 la usan para el cierre de COA y FOA, además este último describe su anatomía detallada, irrigación sanguínea y técnica quirúrgica. Neder en 1983 describe su epitelización. Hudson en 1995 la usa como coadyuvante en el manejo de paladar hendido y Zhao en 1998 reporta su uso en el manejo primario en la reparación de las hendiduras palatinas $(28,29)$.

Se atribuye dentro de las funciones de las bolsas adiposa de Bichat está en la prevención de presión negativa en los neonatos durante la succión, separar los músculos de la masticación entre si y de las estructuras óseas adyacentes, aumentar el movimiento intermuscular y protección de los paquetes neurovasculares (31). Ranke 1884 reporta que la bolsa adiposa tiene un mecanismo de liposis propio diferente al del tejido graso subcutáneo $(28,29)$.

Este colgajo ha sido reportado para el manejo de reconstrucción de defectos de la región palatina, mucosa bucal, cierre de COA-FOA, cobertura de injertos óseos en elevaciones de seno maxilar, relleno en la ATM y reconstrucción de defectos pos- traumático y pos-resecciones en la región maxilar. Con respecto al manejo de las COA y FOA, este colgajo es utilizado principalmente en el manejo secundario de FOA donde hay presencia de tejido fibroso de cicatrización, ausencia de tejido local virgen y/o recurrencia posterior al manejo primario con otros colgajos locales. Aunque también se ha reportado su uso en el manejo primario de COA posterior a procedimientos quirúrgicos orales menores. Se ha sugerido su uso en defectos de tamaño mediano hasta $2 \mathrm{~cm}$ en donde se ha reportado su mayor tasa de éxito $(12,29-32)$.

La ventaja y el éxito de este colgajo radican en el reporte de la no pérdida del surco vestibular, facilidad de realización y su alta vascularización dada por las arterias bucal, temporales profunda anterior y posterior, ramas de la arteria facial y la arteria transversa facial $(1,5,31)$. Sus características anatómicas constituidas por Tideman en 1986 establece que la bolsa adiposa consta de un cuerpo y cuatro procesos: El proceso bucal, pterigoideo y temporales superficial y profundo (30). La extensión del proceso bucal es la más larga y superficial, se encuentra medial al musculo masetero y superficial al musculo buccinador y fascia bucofaríngea. Constituye el 30-40\% del peso total de la bolsa adiposa de Bichat. Su volumen y tamaño son usualmente estables durante toda la vida. Este proceso bucal es libre y por ende puede ser extraído fácilmente a través de una incisión intraoral (30). Por dichas características este proceso bucal es el utilizado en el manejo de las COA-FOA, aunque cabe destacar que también se ha utilizado el proceso del cuerpo y temporal para el manejo del relleno de la ATM (33).

Con respecto a la técnica quirúrgica este colgajo se hace preferiblemente bajo anestesia general, se inicia con infiltración submucosa en la región del paladar donde está el defecto con lidocaína 2\%con epinefrina y se hace incisión perifistular para levantamiento de un colgajo marginal lo suficientemente extenso, libre de tensión para hacer el cierre de la capa mucosa 
nasal o sinusal. Luego se realiza una incisión de $1 \mathrm{~cm}$ en longitud en el fondo del surco gingival vestibular sobre el segundo molar superior y anterior al ducto de salida del conducto Stenon. Se hace disección de las fibras del musculo buccinador y fascia bucofaríngea y luego presión sobre la región inferior al arco cigomático para ayudar a la extrusión de la bolsa adiposa Bichat en su proceso bucal. Luego se hace el movimiento de avance del colgajo hacia el defecto por detrás del último molar y se hace su fijación con sutura absorbible sobre el defecto. En casos donde sea difícil estabilizar el colgajo se hace una sutura del pedículo del colgajo a la mucosa de la región de la tuberosidad y el área retromolar. Si es imposible hacer el posicionamiento del colgajo detrás del último molar y tiene que pasar sobre los dientes, se usa un aparato bucal para prevenir el cierre completo y así evitar el daño del pedículo del colgajo. (30)

Con respecto al manejo posoperatorio se ordena dieta líquida por 1 semana e irrigación con solución salina durante varias veces al día hasta la epitelización del colgajo. Prescripción antibiótica profiláctica con amoxicilina/clavunalato por 5 días y controles semanales hasta la epitelización completa del colgajo con epitelio estratificado escamoso que migra de la encía marginal, esto ocurre alrededor de la 2-6 semana $(30,34)$.

Dentro de los efectos adversos más importante se encuentra la posible asimetría que puede hacer la extrusión de la bolsa adiposa unilateral sin embargo los estudios lo establecen como mínimo e imperceptible (29), también se ha reportado hematoma, infección, lesión del nervio facial, sangrado arterial, perdida del pedículo, limitación apertura oral sin embargo teniendo en cuenta las recomendaciones y limitaciones, la aplicación del colgajo de bolsa adiposa Bichat es un procedimiento seguro y exitoso (27).

\section{Colgajos pediculados}

\section{El colgajo de lengua}

Introducido por Lexter 1909 para reconstrucciones intraorales (35). Actualmente son utilizados para el cierre de COA Y FOA resultado de heridas por arma de fuego, resección de lesiones neoplásicas, secuelas de labio y paladar hendido y paladar hendido, secuela de procesos infecciosos (36), y para asegurar cobertura de injertos óseos (37). Además se han utilizado recientemente para la reconstrucciones labiales $(38,39)$. Sus diferentes variantes datan desde 1956 donde Klopp y Schurter describen el colgajo de lengua pediculado lateral para cierre de defecto palatino posterior a ablación de lesión cancerígena. En 1957 Conley propone le uso del colgajo de lengua para cobertura temporal de heridas o reconstrucciones definitivas (35). Guerrero-santos y Altamirano en 1966 usan el colgajo de lengua dorsal de base anterior para el cierre de paladar hendido. En 1972 Cadena y col. describen el rico plexo vascular submucoso de la lengua y demostraron que los colgajos de lengua podrían ser usados en cualquier dirección, incluso con un grosor de tan solo 3mm. (40) En 1977 Hokcstein y en 1980 Carreirao y Lessa reportan el uso de colgajos de lengua largos de espesor completo con resultados favorables. En 1979 Sachs utiliza un colgajo de lengua dorsal de base posterior para el cierre de una fistula oroantral y finalmente en 1989 Busic y en 1992 Assuncao utilizan colgajos delgados, demostrando su versatilidad y seguridad para el cierre de fistulas palatinas(40). Sin embargo es Bracka en 1981 quien da las bases anatómicas para la realización el colgajo de lengua, en donde establece la irrigación del colgajo dado por las arterias raninas en la zona anterior y por las arterias dorsales en la zona posterior, la longitud puede llegar de $5-6 \mathrm{~cm}$ teniendo como límite posterior las papilas gustativas mayores, el ancho del colgajo oscila entre $2.5-3 \mathrm{~cm}$ o ser $20 \%$ mayor al defecto, su grosor debe ser de 5-6mm para tomar algo de musculo longitudinal de la lengua y así proteger el plexo vascular submucoso.

Aunque se ha descrito diferentes diseños del colgajo de lengua como los laterales y ventrales (36), el colgajo utilizado para el cierre de COA o FOA es el de cara dorsal de base posterior o anterior según la necesidad (32). Con respecto a la técnica quirúrgica para el manejo de una FOA secundaria la cual realiza bajo anestesia general y respetando los principios anatómicos descritos por Bracka se inicia con la infiltración submucosa con lidocaína al $2 \%$ con epinefrina, se realiza una incisión perifistular, se diseca cuidadosamente el colgajo resultante o marginal y se invierten los márgenes suturándolos con vycril 4/0 sin tensión, conformado de esta manera el plano sinusal o nasal. A continuación se realiza la colocación de 6 suturas de vicryl 3/0 sin retirar sus agujas en los márgenes mucosos circundantes a la FOA, y luego se reparan de forma extraoral con una pinza; esto con el objetivo de facilitar posteriormente una adecuada sutura del colgajo 
lingual. Luego se procede a la colocación de un punto de sutura con seda 0 o 2/0 de forma transversal en la punta lingual para permitir la tracción y exposición de la cara ventral lingual, se realiza infiltración submucosa sobre sitio donante con lidocaína $2 \%$ con epinefrina. Se marca con azul de metileno la longitud del colgajo que será entre 5-6cm para permitir un adecuado rango de movilidad de la lengua durante la cicatrización y el ancho del colgajo el cual será los necesarios para cubrir más de un $20 \%$ de la FOA. La incisión y disección del colgajo lingual es realizada con hoja de bisturí \#15. El grosor del colgajo estará entre $3 \mathrm{~mm}-1 \mathrm{~cm}$ con el objetivo de asegurar la irrigación submucosa muscular. Debe evitarse el uso de electrocauterio, con el fin de no comprometer la vascularización en los márgenes del colgajo y del lecho receptor. El sitio donante es cerrado con vicryl 3-0. Se determina la posición del colgajo y las suturas previamente colocadas pasan ahora el colgajo lingual y se busca un afrontamiento adecuado sobre lecho receptor. Una vez realizado lo anterior se anudan las suturas y los márgenes restantes se cierran con vicryl 3/0. Finalmente se observa el color del colgajo para determinar la correcta vascularización y no sitios sangrantes.

Se deja por periodo de 3 semanas donde se realiza prueba de isquemia sobre el colgajo y se determina la viabilidad del mismo. Si hay adecuado adhesión del mismo se procede hacer su liberación del pedículo con anestesia local.

Este colgajo se ha usado para manejo primario de defectos entre de $2-3 \mathrm{~cm}$ donde los colgajos locales sean insuficientes para su cierre o manejo secundario (incluyendo el uso de colgajo marginal para conformación del piso de seno o fosa nasal según sea el caso) de lesiones recidivantes manejadas con técnicas más conservadoras primariamente. $(40,41)$

A pesar de las grandes ventajas que se muestran del colgajo de lengua también se han descrito varias desventajas como el hecho de que su realización sea bajo anestesia general, la posibilidad de desgarro del colgajo con pérdida de vascularización, necrosis del colgajo, uso de fijación intermaxilar o eventual episodio de hemorragia. Además siempre ha quedado la inquietud con respecto a las posibles alteraciones que este colgajo puede producir sobre el lenguaje, la función masticatoria y la competencia velo faríngea, sin embargo hasta el momento no se ha reportado ninguna de forma permanente(5, 8, 42,43).

\section{Colgajo de buccinador}

En 1975 Kaplan utilizó un colgajo de mucosa bucal para la reconstrucción del paladar blando. En 1987 Maeda mejora la vascularización de la mucosa utiliza fibras del musculo buccinador para la reparación del paladar hendido. Bozola en 1989 describe la arteria bucal como el principal pedículo para el musculo buccinador. Cartens en 1991 describe el colgajo miomucoso en isla de músculo buccinador pediculado a la arteria y la vena facial, afirmando que el mayor aporte sanguíneo del musculo provenía de la arteria facial. Zhao en 1999 describe el colgajo en isla miomucoso de buccinador el cual difiere de las otras técnicas en su tunelización a través del margen mandibular inferior, el cual le confiere las ventajas de una cantidad considerable de tejido, el remplazo de mucosa por mucosa, la no necesidad de una incisión externa, reducción de la morbilidad del sitio donante y resultados cosméticos y funcionales óptimos $(44,45)$.

Aunque el colgajo miomucoso de musculo buccinador con sus diferentes técnicas se ha reportado para el manejo paladar hendido, reconstrucciones labiales, piso de boca, lengua, nasales y paladar de $1-8 \mathrm{~cm}$, para el manejo de la COA y FOA se constituye como una opción terciaria debido a su técnica quirúrgica compleja basada en los reparos anatómicos adyacentes a su toma. Aparte de la dificultad de la técnica mostrando otras desventajas como lesión al nervio marginal mandibular, perdida del pedículo por interposición interdental, infección y hematoma (44-56).

\section{Colgajo de músculo temporal}

Descrito en 1872 por Verneuil posterior a una artroplastia de la ATM, en 1889 Wagner describe un colgajo osteogaleal usando como pedículo las arterias temporales profundas, en 1912 Murphy lo utiliza como colgajo de interposición posterior a una artroplastia de atm. En 1963 Bakamjian reporta el uso del colgajo musculo temporal para la reconstrucción de defectos que incluyan una parte o toda la hemimaxila. De ahí en adelante se han publicado muchos artículos donde se reporta su uso para anquilosis ATM, remplazo del disco ATM, reanimación facial, reconstrucción palatina posterior a resecciones oncológicas, reconstrucciones de hendiduras palatinas congénitas recidivantes, reconstrucción de región cigomática hipoplasica o ausente en síndrome treacher collins, obliteración orbitaria pos exanteración, restauración 
del parpado en lagoftalmías, obliteraciones del seno frontal en fracturas o infecciones, reconstrucción de base de cráneo y reconstrucción de la mucosa oral y faríngea en ablaciones tumorales o avulsiones traumáticas (57).

Su obtención dependerá de los principios quirúrgicos determinados por la anatomía concerniente a la región preauricular y temporal en donde se enfocara en mantener indemnes estructura anatómicas como las ramas del nervio facial y las arterias temporales profundas y la temporal superficial, y sobre la cantidad del defecto y región anatómica en donde se utilizara $(57,58)$.

Aunque realmente es una opción terciaria en el manejo de defectos que involucren la región maxilar y su uso haya sido desplazado gradualmente por los colgajos libres vascularizado, puede ser la primera opción en caso de pacientes con defectos mayores de $3 \mathrm{~cm}$ y que por su estado de salud como cardiopatías impidan estar bajo un tiempo quirúrgico prolongado con anestesia general o que sea candidato a sometimiento posterior a quimioterapia, en donde no será viable la utilización de un colgajo libre microvascularizado por su riesgo de pérdida $(4,57,58)$.

\section{Conclusión}

La revisión presentada muestra de forma básica y especifica las diferentes opciones quirúrgicas para el manejo de las COA Y FOA, las cuales al utilizarlas hay que tener en consideración las características de la lesión, consideraciones inherentes a la técnica quirúrgica, ventajas y desventajas e indicaciones de cada de una de ellas con el objetivo de obtener los mejores resultados pos operatorios.

\section{Bibliografía}

1. Abuabara et al. Evaluation of different treatments for oroantral/ oronasal communications: experience of 112 cases. int. j. oral maxillofac. surg. 2006; 35: 155-158.

2. Itzhak Brook. Sinusitis of odontogenic origin. otolaryngologyhead and neck surgery (2006) 135, 349-355.

3. Andric et al.functional endoscopic sinus surgery as an adjunctive treatment for closure of oroantral fistulae: a retrospective analysis. oral surg oral med oral pathol oral radiol endod 2010;109:510-516.

4. Ehrl et al. oroantral communication. Epicritical study of 175 patients, with special concern to secondary operative closure. int j oral srg. 1980: 9: 351-358.

5. Lazow et al. surgical management of the oroantral fistula: flap procedures. operative techniques in otolaryngology--head and neck surgery, vol 10, no 2 (jun), 1999: pp 148-152.
6. Visscher, van Minnen, and Bos. Closure of Oroantral Communications. J Oral Maxillofac Surg 2010.68:1384-1391.

7. Fickling, Oral surgery involving the maxillary sinus.Charles Tomes Lecture delivered at the Royal College of Surgeons of England on 20th July 1956.

8. Lehman et al, closure of anterior palate fistulae. Cleft palate journal, January 1978, Vol n 15 No 1.

9. Kale et al. Treatment of oroantral fistula using palatal flap: a case report and technical note. J. Int Oral Health 2010, Volume 2 (Issue 3).

10. Watzak et al. Bony Press-Fit Closure of Oro-Antral Fistulas. J Oral Maxillofac Surg 63: 1288-1294,2005.

11. Majid. Persistent oronasal after primary management of facial gunshot injuries. British Journal of Oral and Maxillofacial Surgery 46 (2008) 50-52.

12. Visscher, van Minnen. Closure of oroantral communications using biodegradable polyurethane foam: a feasibility study. $J$ Oral Maxillofac Surg 2010. 68:281-286.

13. Cohen et al. Cleft palate fistulas a multivariate statistical analysis of prevalence, etiology, and surgical management. Vol 87 n 61991.

14. Lee at al. Repair of oroantral communications in the third molar region by random palatal flap. Int. J. Oral Maxillofac. Surg. 2002; 31: 677-680.

15. Costa et al. Endoscopic Surgery for Maxillary Sinusitis.J Oral Maxillofac Surg 65:223-228, 2007.

16. Hori et al. Application of the interseptal alveolotomy for closing the oroantral fistula. J. Oral Maxillofac. Surg 53: 1392-1396, 1995.

17. Hass et al. A preliminary study of monocortical bone grafts for oroantral fistula closure. Oral Surg Oral Med Oral Pathol Oral Radiol Endod 2003;96:263-6.

18. Isler et al. Closure of oroantral fistula using auricular cartilage a new method to repair an oroantral fistula. British Journal Of oral a maxillofacial surgery (2011) e1-e2.

19. Stetner et al. Metal plates and foils for closure of oroantral fistulae. J Oral Maxillofac Surg. 66: 1551-1555, 2008.

20. Gacic et al. The closure of oroantral communications with resorbable PLGA-coated-TCP root analogs, hemostatic gauze, or buccal flaps A prospective study. Oral Surg Oral Med Oral Pathol Oral Radiol Endod 2009;108:844-850.

21. Ogunsalu C. A new surgical management for oro-antral communication. West Indian Med J 2005; 54 (4): 261.

22. Silver et al. Preparation and use of fibrin glue in surgery. Biomaterials 16 (1995) 891-903.

23. Kitagawa. Use of third molar transplantation for closure of the oroantral conmmunication after tooth extraction: A report of 2 cases. Oral Surg Oral Med Oral Pathol Oral Radiol Endod 2003;95:409-15.

24. Marzola C. Surgical and medicines treatment of buccal sinuses communications.

25. Lehman JR. Closure of palatal fistulas. Operative Techniques in Plastic and Reconstructive Surgery, Vol 2, No 4 (November), 1995: pp 255-262.

26. Salins et al. Anteriorly based palatal flap for closure of large oroantral fistula. Oral Surg Oral Med Oral Pathol Oral Radiol Endod 1996:82;253-56.

27. Anavi et al. Palatal rotation-advancement flap for delayed repair of oroantral fistula: A retrospective evaluation of 63 cases. Oral Surg Oral Med Oral Pathol Oral Radiol Endod 2003;96;527-34.

28. Grobe et al. The use of buccal fat pad (BFP) as pedicle graft in cleft palate surgery Int. J. Oral Maxillofac. Surg. 2011. 


\section{Galvis Aparicio A.}

29. Singh et al. Buccal pad of fat and its applications in oral and maxillofacial surgery: a review of published literature 20042009. Oral Surg Oral Med Oral Pathol Oral Radiol Endod 2010.

30. Ashtiani et al.Closure of palatal fistula with buccal fat pad flap. J Oral Maxillofac Surg 2011; 40:250-254.

31. Poeschl. Closure of oroantral communications with bichat's buccal fat pad. J Oral Maxillofac Surg 67:1460-1466, 2009.

32. Hassani et al. Measurement of volume changes of sinus floor augmentation covered with buccal fat pad: a case series study. Oral Surg Oral Med Oral Pathol Oral Radiol Endod 2009;107:369-374.

33. Singh et al. Retrospective analysis of use of buccal fat pad as an interpositional graft in tempormandibular joint ankylosis: preliminary study. J Oral Maxillofac Surg, 2011.

34. Hanazawa et al. Closure of oroantral communications using a pedicle buccal fat pad graft. American association of oral and maxillofacial surgeons, 1995.

35. Komisar A. The applications of tongue flaps in head and neck surgery. Head and neck surgery. Vol. 62, No 8, October 1986

36. Kim et al. Use of the tongue flap for intraoral reconstruction: a report of 16 cases. J Oral Maxillofac Surg 56:716-719, 1998

37. Sandor et al. Dental implants placed into alveolar clefts reconstructed with tongue flaps and bone grafts. Oral Surg Oral Med Oral Pathol Oral Radiol Endod 2010:109;e1-e7

38. Wushou et al. Reconstruction of defects in the lip vermillion with bilateral tongue flaps and review of the literature. Asian journal of oral and maxillofacial surgery 22 (2010), 99-101.

39. Yano et al. Combined tongue flap and V-Y advancement flap for lower lip defects. British journal of plastic surgery 2005 58, 258-262.

40. Alcoforado A. The design of tongue flaps for the closure of palatal fistulas. Plastic and reconstructive surgery 1993.

41. Guerrero-santos y Ferndandez. Further experience with tongue flap in cleft palate repair. Plastic and reconstructive surgery, University of Guadalajara.

42. Sendota et al. Cierre de fistulas palatinas amplias mediante colgajos de lengua en pacientes con secuelas de paladar hendido. Revista odontológica mexicana. Vol 10. Num 32006 131-137.

43. Elyassi et al. Closure of difficult palatal fistulas using a "parachuting and anchoring" technique with the tongue flap.Oral Surg Oral Med Oral Pathol Oral Radiol Endod 2011.
44. Rattan V. A simple technique for use of buccal pad of fat in Temporomandibular joins reconstruction. J Oral Maxillofac Surg 64: 1447-1451, 2006.

45. González et al. Colgajo miomucoso de buccinador en la reconstrucción de defectos del área maxillofacial. Rev Esp Cir Oral y Maxilofac 2005, 27, 4:197-205.

46. Ferri et al. A novel technique for cheek mucosa defect reconstruction using a pedicled buccal fat pad and buccinator myomucosal island flap. Oral oncology 2009 45, 59-62.

47. Ferri et al. Donor site morbidity using buccinators myomucosal island flap. Oral Surg Oral Med Oral Pathol Oral Radiol Endod 2011;111:306-311.

48. Fosko et al. Nasal reconstruction with the cheek island pedicled flap. J Am Acad Dermatol 1996;35:508-7.

49. Bozola et al. Partial buccinators myomucosal flap posteriorly based. 1995

50. Ferrari et al. Reconstruction large palate defects: The double buccinator myomucosal island flap. J Oral Maxillofac Surg 68:924-926, 2010.

51. Bianchi et al. Myomucosal cheek flaps: applications in intraoral reconstruction using three different techniques. Oral Surg Oral Med Oral Pathol Oral Radiol Endod 2009;108:353-359

52. Maeda et al. A t-shaped musculomucosal buccal flap method for cleft palate surgery. Plastic and reconstructive surgery, june 1987.

53. Massarelli et al. Three-dimensional primary reconstruction of anterior mouth floor and ventral tongue using the trilobed buccinators myomucosal island flap. J Oral Maxillofac Surg 2008; 37: 917-922.

54. Zhao et al. The buccinators musculomucosal island flap for partial tongue reconstruction. J Am Coll Surg 2003; 196:753-760.

55. Ferrari et al. Buccinator myomucosal island flap for reconstruction of the floor of the mouth. J Oral Maxillofac Surg 66:394-400, 2008.

56. Reid et al. The anatomy of buccinators-insights from functional casts of the oral vestibule. Archives of oral biology 200348 , 787-795.

57. Dallan et al. Temporalis myofascial flap in maxillary reconstruction: anatomical study and clinical application. Journal of cranio-maxillofacial surgery 2009 37, 96-101.

58. Rodríguez et al.utilización del colgajo de musculo temporal en cirujareconstructiva maxillofacial. Revisión de 104 casos. Res Esp Ciru Oral Y maxilofac 2004;26:228-237. 